\title{
SHORT CHAINS AND REGULAR COMPONENTS
}

\author{
IDUN REITEN, ANDRZEJ SKOWROŃSKI, AND SVERRE O. SMALØ
}

(Communicated by Maurice Auslander)

\begin{abstract}
Let $\Lambda$ be a finite-dimensional $k$-algebra with $k$ an algebraically closed field and ind $\Lambda$ a chosen subcategory of a complete set of isomorphism classes of finitely generated indecomposable $\Lambda$-modules. This paper deals with the regular components of ind $\Lambda$ consisting of modules that are not the middle of any short chain. It is proved that the number of such components containing only a finite number of $D T r$-orbits is finite. Further, the infinite radical of such a component is zero and the component is isomorphic to the mesh category of its underlying translation quiver. Families of selfinjective algebras having such components are constructed.
\end{abstract}

\section{INTRODUCTION}

Let $\Lambda$ be an artin algebra. A $\Lambda$-module $C$ is said to be the middle of a short chain if there exists an indecomposable $\Lambda$-module $X$ with $\operatorname{Hom}_{\Lambda}(X, C) \neq 0$ and $\operatorname{Hom}_{\Lambda}(C, D \operatorname{Tr} X) \neq 0$ [AR]. Any directing module $C$, that is an indecomposable module $C$ such that there is no proper chain of nonzero nonisomorphisms between indecomposable modules from $C$ to $C$, is not the middle of a short chain, and there are also many other examples. In fact, being the middle of a short chain is closely related to lying on a short cycle $C \rightarrow^{f} X \rightarrow^{g} C$ where $X$ is indecomposable and $f$ and $g$ are nonzero nonisomorphism [RSS].

Modules that are not the middle of a short chain came up in connection with finding sufficient conditions for indecomposable modules to be determined by their composition factors, generalizing earlier results about directing modules [AR]. This is one reason it is of interest to investigate when modules are not the middle of short chains.

In general, directing modules have played an important role in representation theory, and results about directing modules serve as an inspiration for what might be true for modules that are not the middle of short chains. In this

Received by the editors June 20,1991; the contents of this paper were presented at the Oberwolfach meeting on Representation Theory of Algebras, May 1991, at the Warwick Symposium on Ring Theory and its Applications, July 1991, and at the RIMS Symposium Representation Theory of Finite Groups and Finite Dimensional Algebras, Kyoto, October 1991.

1991 Mathematics Subject Classification. Primary 16G10, 16G20, 16G70, 16D90; Secondary 16E30, 16S90, 18A32.

Partially supported by the Norwegian Research Council of Science and Humanities. This work was done while the second author visited Trondheim.. 
connection it is also of interest to investigate the relationship between modules that are not the middle of a short chain and directing modules.

For algebras of finite representation type there are many cases when all indecomposable modules are not the middle of a short chain. In fact, if $\Lambda$ is an algebra over an algebraically closed field $k$, there is always a finite covering of $\Lambda$ having this property [AR]. For finite representation type we also have a nice relationship to directing modules, since an indecomposable module that is not the middle of a short chain is directing if it is sincere [RSS].

By a component $\mathscr{C}$ for $\Lambda$ we mean a subcategory of the category ind $\Lambda$ of indecomposable $\Lambda$-modules closed under irreducible maps and connected modulo the infinite radical. Associated with $\mathscr{C}$ is its $A R$-quiver, which is a component of the $A R$-quiver for $\Lambda$. In this paper we investigate when all modules in a component $\mathscr{C}$ for an artin algebra $\Lambda$ are not the middle of a short chain. We assume that $\Lambda$ is an algebra over an algebraically closed field, and we here restrict our attention to regular components, that is, components containing neither projective nor injective modules. We show that when the number of $D T r$-orbits is finite, $\Lambda /$ ann $\mathscr{C}$ must be a tilted algebra via a regular tilting module and $\mathscr{C}$ is the connecting component of $\Lambda /$ ann $\mathscr{C}$. In particular, since a connecting component of a tilted algebra always consists of directing modules, all modules in $\mathscr{C}$ are directing as $\Lambda /$ ann $\mathscr{C}$-modules. Moreover, we show that $\Lambda$ can have only a finite number of components with a finite number of DTr-orbits and consisting entirely of modules that are not the middle of a short chain.

Regular components $\mathscr{C}$ consisting entirely of directing modules were described in [SkSm] as the connecting components of tilted algebras via regular tilting modules. Our main result is a generalization to modules not being the middle of a short chain. It is, however, an open question whether there can be regular components with all modules not the middle of a short chain having an infinite number of $D T r$-orbits. This cannot happen if all the modules are directing [SkSm].

In $\S 1$ we prove our main result on regular components where all modules are not the middle of a short chain. In $\S 2$ we show that for any finite quiver $\Delta$ that is neither Dynkin nor Euclidean with no oriented cycle and at least three vertices, there is always some (self-injective) algebra $\Lambda$ with a regular component of type $\mathbb{Z} \Delta$ such that all modules are not the middle of a short chain. It is easy to see that self-injective algebras do not have indecomposable modules that are directing.

All modules will be finitely generated right modules, and we denote $\operatorname{Hom}_{\Lambda}(M, N)$ by $(M, N)$.

\section{SHORT CHAINS AND REgULAR COMPONENTS}

Let $\Lambda$ be an algebra over an algebraically closed field $k$. If $\Lambda$ is a tilted algebra then $\Lambda$ has a regular connecting component if and only if $\Lambda$ is a tilted algebra via a regular tilting module [R2, R3]. When $\Lambda$ is a tilted algebra via a regular tilting module, $\Lambda$ has a unique connecting component, which consists entirely of directing modules [R2]. The $A R$-quivers of these components are the $\mathbb{Z} \Delta$ where $\Delta$ is a finite quiver having no oriented cycles and at least three vertices and is neither of Dynkin nor Euclidean type [R2]. These are exactly 
the $A R$-quivers occurring for regular components consisting entirely of directing modules [SkSm]. We show that the same $A R$-quivers occur for components $\mathscr{C}$ consisting entirely of modules that are not the middle of a short chain and having only a finite number of $D T r$-orbits. In fact we show that if $\Lambda$ has such a component $\mathscr{C}$, then the algebra $\Lambda / \operatorname{ann} \mathscr{C}$, where ann $\mathscr{C}$ denotes the annihilator of the modules in $\mathscr{C}$, is a tilted algebra via a regular tilting module and $\mathscr{C}$ is the connecting component of $\Lambda /$ ann $\mathscr{C}$. We also show that $\Lambda$ can have only a finite number of such components.

We first prove a series of preliminary results, most of which hold for arbitrary artin algebras. So assume throughout this section that $\Lambda$ is an artin algebra and $\mathscr{C}$ is a regular component for $\Lambda$ with a finite number of $D T r$-orbits.

Lemma 1.1. The AR-quiver of the component $\mathscr{C}$ is $\mathbb{Z} \Delta$ for some finite valued quiver $\Delta$ without oriented cycles.

Proof. Since $\mathscr{C}$ contains no projective modules, $\mathscr{C}$ must be an infinite component [A]. Using that $\mathscr{C}$ has only a finite number of $D T r$-orbits, it follows that not all modules in $\mathscr{C}$ are periodic, and consequently they are all nonperiodic. We then use that any regular component with no $D T r$-periodic module has an $A R$-quiver of the form $\mathbb{Z} \Delta$ for some valued quiver $\Delta$ without oriented cycles [Z] (see also [L]). In this case $\Delta$ is clearly finite.

Lemma 1.2. Let the AR-quiver $\mathscr{C}$ be $\mathbb{Z} \Delta$ as above, and let $M$ be the direct sum of the indecomposable modules corresponding to the vertices of a fixed $\Delta$. Then $M$ is a faithful $\Lambda /$ ann $\mathscr{C}$-module.

Proof. Denote by $\mathscr{D}$ the modules on $\Delta$ and their successors and by $\mathscr{E}$ the modules on $\Delta$ and their predecessors. Since $\Lambda$ is artin, there is some $\Lambda$-module $N$ that is a finite direct sum of modules in $\mathscr{D}$ such that ann $\mathscr{D}=\operatorname{ann} N$. Hence there is a monomorphism $\Lambda / \operatorname{ann} N \rightarrow N^{n}$ for some $n \geq 1$. Let $M$ be the direct sum of the indecomposable modules corresponding to the vertices of $\Delta$. We want to show that $\Lambda /$ ann $N \rightarrow N^{n}$ factors through some module in add $M$, so that ann $M=\operatorname{ann} N=\operatorname{ann} \mathscr{D}$.

Denote by $F$ the subfunctor of $(, N)$ generated by all images of morphisms from $(, M)$ to $(, N)$. Then it is easy to see that $(, N) / F$ vanishes on projectives and, in fact, vanishes on all but a finite number of indecomposable modules. Hence any map $\Lambda \rightarrow N$ factors through some $M^{t}$ and, therefore, ann $M \subseteq$ ann $N$. Since $M$ is a direct sum of modules in $\mathscr{D}$, we then have ann $M=\operatorname{ann} N$. Similarly we show ann $\mathscr{E}=$ ann $M$, so that $M$ is a faithful $\Lambda /$ ann $\mathscr{C}$-module.

Now let $\Lambda$ be an algebra over an algebraically closed field $k$, let $\mathscr{D}$ be a component of $\Lambda$, and let $\Sigma$ be the $A R$-quiver of $\mathscr{D}$. Recall that the mesh category $k(\Sigma)$ of $\Sigma$ is the path category $k[\Sigma]$ of $\Sigma$ over $k$ modulo the relations given by the meshes corresponding to almost split sequences. The component $\mathscr{D}$ is said to be standard if $\mathscr{D}$ is equivalent to the mesh category $k(\Sigma)$. For a component $\mathscr{C}$ of $\Lambda$ with $A R$-quiver $\mathbb{Z} \Delta$ where $\Delta$ is a finite quiver with no oriented cycles, fix a vertex $x$ and let $X$ be the corresponding $\Lambda$-module. The full subtranslation quiver $\Gamma$ of $\mathbb{Z} \Delta$ whose vertices are the predecessors of $x$ is the $A R$-quiver of the preinjective component of a hereditary path algebra $k \tilde{\Delta}$, where $\tilde{\Delta}$ has the same underlying graph as $\Delta$. 
Denote by $d_{x}$ the function on $\mathbb{Z} \Delta$ given by

$$
d_{x}(y)=\operatorname{dim}_{k}(Y, X) / \operatorname{rad}^{\infty}(Y, X),
$$

where $Y$ is the $\Lambda$-module corresponding to $y$. Further let $\tilde{X}$ be the injective $k \tilde{\Delta}$-module corresponding to $x$, and define the function $\tilde{d}_{x}$ on $\Gamma$ by $\tilde{d}_{x}(y)=\operatorname{dim}_{k}(\tilde{Y}, \tilde{X})$, where $\widetilde{Y}$ is the preinjective $k \widetilde{\Delta}$-module corresponding to $y$. Finally define $\overline{d_{x}}(y)=\operatorname{dim}_{k(\mathbb{Z} \Delta)}(y, x)$. We then have

Lemma 1.3. Let $\Lambda$ be a finite-dimensional algebra over an algebraically closed field $k$, and let $\mathscr{C}$ be a component of $\Lambda$ with AR-quiver $\mathbb{Z} \Delta$ where $\Delta$ is a finite quiver with no oriented cycles. Fix a vertex $x$ of $\mathbb{Z} \Delta$ and let $X$ be the corresponding $\Lambda$-module. Let the translation quiver $\Gamma$, the hereditary algebra $k \tilde{\Delta}$, and the functions $d_{x}, \tilde{d}_{x}$, and $\bar{d}_{x}$ be as above.

(a) The functions $d_{x}, \tilde{d}_{x}$, and $\overline{d_{x}}$ coincide on $\Gamma$, and $d_{x}$ and $\overline{d_{x}}$ coincide on $\mathbb{Z} \Delta$.

(b) There is only a finite number of $\Lambda$-modules $Y$ corresponding to predecessors of $X$ such that $\operatorname{Hom}_{\Lambda}(Y, X)=0$.

(c) The mesh category $k(\mathbb{Z} \Delta)$ is equivalent to $\mathscr{C} / \operatorname{rad}^{\infty} \mathscr{C}$.

Proof. (a) From [IT] combined with the snake lemma and using duality we have for each natural number $n$ and each almost split sequence $0 \rightarrow D \operatorname{Tr} Y \rightarrow E \rightarrow$ $Y \rightarrow 0$, the exact sequence of functors

$$
\begin{aligned}
0 & \rightarrow(Y,) / \operatorname{rad}^{n}(Y,) \rightarrow(E,) / \operatorname{rad}^{n+1}(E,) \\
& \rightarrow \operatorname{rad}(D T r Y,) / \operatorname{rad}^{n+2}(D T r Y,) \rightarrow 0 .
\end{aligned}
$$

Since for each $Z$ and $A$ in $\bmod \Lambda$ there exists a natural number $m$ with $\operatorname{rad}^{m}(Z, A)=\operatorname{rad}^{\infty}(Z, A)$, we obtain from these exact sequences the exact sequence

$$
\begin{aligned}
0 & \rightarrow(Y,) / \operatorname{rad}^{\infty}(Y,) \rightarrow(E,) / \operatorname{rad}^{\infty}(E,) \\
& \rightarrow \operatorname{rad}(D T r Y,) / \operatorname{rad}^{\infty}(D T r Y,) \rightarrow 0
\end{aligned}
$$

of functors on $\bmod \Lambda$.

Since the translation quiver $\mathbb{Z} \Delta$ has no oriented cycles and only a finite number of orbits under translation, there exists for each pair $y, x$ of vertices in $\mathbb{Z} \Delta$ a number $n_{y, x}$ such that there are no chains of arrows in $\mathbb{Z} \Delta$ of length greater than $n_{y, x}$ starting in $y$ and ending in $x$. Now fix an $X$ in $\mathscr{C}$ and let $x$ be the corresponding vertex in $\mathbb{Z} \Delta$. By using the exact sequence of functors above we may calculate $\operatorname{dim}_{k}\left((Y, X) / \operatorname{rad}^{\infty}(Y, X)\right)$ by induction on $n_{y, x}$ where $y$ is the vertex in $\mathbb{Z} \Delta$ corresponding to the module $Y$. The base of this induction is the fact that $\operatorname{dim}_{k}(Y, X) / \operatorname{rad}^{\infty}(Y, X)=0$ if $n_{y, x}=0$ and $X \not Y$ and that $\operatorname{dim}_{k}\left((X, X) / \operatorname{rad}^{\infty}(X, X)\right)=1$. Now the function $d_{x}(y)=\operatorname{dim}_{k}\left((Y, X) / \operatorname{rad}^{\infty}(Y, X)\right)$ is completely determined by $\mathbb{Z} \Delta$.

If $I \neq \widetilde{X}$ is an indecomposable injective $k \widetilde{\Delta}$-module, then $I$ is not simple and the epimorphism $I \rightarrow I / \operatorname{soc} I$ is minimal left almost split. Then we have an isomorphism $(I / \operatorname{soc} I, \tilde{X}) \underset{\rightarrow}{\rightarrow}(I, \tilde{X})$. If $0 \rightarrow A \rightarrow B \rightarrow C \rightarrow 0$ is an almost split sequence of preinjective $k \widetilde{\Delta}$-modules then $0 \rightarrow(C, \tilde{X}) \rightarrow(B, \tilde{X}) \rightarrow$ $(A, \tilde{X}) \rightarrow 0$ is exact. From this it follows that $d_{x}$ and $\tilde{d}_{x}$ coincide on $\Gamma$. 
Since the preinjective component $\mathscr{C}$ of $k \tilde{\Delta}$ is known to be standard (see [R1, p. 63]), it follows that $\tilde{d}_{x}=\overline{d_{x}}$ on $\Gamma$. Then we clearly have $d_{x}=\overline{d_{x}}$ on $\mathbb{Z} \Delta$.

(b) It is well known that all but a finite number of preinjective $k \widetilde{\Delta}$-modules are sincere (see [DR, B]) and hence $\tilde{d}_{x}(y)=0$ for only a finite number of predecessors $y$ of $x$. By (a) we then have $d_{x}(y)=\operatorname{dim}_{k}\left((Y, X) / \operatorname{rad}^{\infty}(Y, X)\right)=0$ for only a finite number of indecomposable $\Lambda$-modules $Y$ corresponding to predecessors of $y$. It follows that $\operatorname{Hom}_{\Lambda}(Y, X)=0$ for only a finite number of such $Y$.

(c) Since $\Delta$ is a finite quiver with no oriented cycles, we can define a full and dense functor from the path category $k[\mathbb{Z} \Delta]$ to $\mathscr{C} / \mathrm{rad}^{\infty} \mathscr{C}$ in such a way that the meshes go to zero. Hence there is induced a functor $F: k(\mathbb{Z} \Delta) \rightarrow$ $\mathscr{C} / \operatorname{rad}^{\infty} \mathscr{C}$. If $x$ is a vertex of $\mathbb{Z} \Delta$, it follows by (a) that $d_{x}(y)=\overline{d_{x}}(y)$ for all $y$ in $\mathbb{Z} \Delta$, so that $F$ is an equivalence of categories.

Lemma 1.4. Let $\Lambda$ be a finite-dimensional algebra over an algebraically closed field. Let $\mathscr{C}$ be a regular component with a finite number of DTr-orbits as before, with AR-quiver $\mathbb{Z} \Delta$ for a finite quiver $\Delta$ without oriented cycles. Assume that all modules in $\mathscr{C}$ are not the middle of a short chain.

Then $\operatorname{rad}^{\infty}(X, Y)=0$ for all $X$ and $Y$ in $\mathscr{C}$, so that $\mathscr{C}$ is standard.

Proof. Let $X$ and $Y$ be in $\mathscr{C}$ and assume that $\operatorname{rad}^{\infty}(X, Y) \neq 0$. Choose a nonzero map $f$ in $\operatorname{rad}^{\infty}(X, Y)$ and let $t_{i}:\left(, K_{i}\right) \rightarrow \operatorname{rad}^{i}(, Y)$ be a projective cover. Then $(, f):(, X) \rightarrow(, Y)$ factors through $t_{i}$. Hence $\operatorname{Hom}_{\Lambda}\left(X, K_{i}\right) \neq 0$, and consequently $\operatorname{Hom}_{\Lambda}\left(X, L_{i}\right) \neq 0$ for an indecomposable summand $L_{i}$ of $K_{i}$. We also have a chain of irreducible maps between indecomposable modules of length $i$ (counting maps) from $L_{i}$ to $Y$. Because $\Delta$ is finite, $L_{i}$ must be a predecessor of $X$ for $i$ large enough. Since by Lemma 1.3 there is only a finite number of predecessors $Z$ of $X$ in $\mathscr{C}$ with $\operatorname{Hom}_{\Lambda}(Z, X)=0$, we get that for $i$ large enough $\operatorname{Hom}_{\Lambda}\left(\operatorname{Tr} D L_{i}, X\right) \neq 0$. This contradicts the fact that $X$ is not the middle of a short chain. Hence we have $\operatorname{rad}^{\infty}(X, Y)=0$ for all $X$ and $Y$ in $\mathscr{C}$. This shows that $\mathscr{C}$ is standard by using Lemma 1.3.

Lemma 1.5. Let $M$ be a faithful module over an artin algebra $\Lambda$ and assume $\operatorname{Hom}_{\Lambda}(M, D T r M)=0$. Then we have $\operatorname{pd}_{\Lambda} M \leq 1$.

Proof. To show $\operatorname{pd}_{\Lambda} M \leq 1$, it is well known that it is sufficient to show $\operatorname{Hom}_{\Lambda}(D \Lambda, D \operatorname{Tr} M)=0$. Since $M$ is faithful, $D M$ is a faithful $\Lambda^{\text {op }}$ module. Hence we have a monomorphism $\Lambda^{\mathrm{op}} \rightarrow(D M)^{n}$ and, therefore, an epimorphism $M^{n} \rightarrow D \Lambda$. It follows that $\operatorname{Hom}_{\Lambda}(D \Lambda, D T r M)=0$ since $\operatorname{Hom}_{\Lambda}(M, D T r M)=0$.

Lemma 1.6. Let $M$ be a faithful module over an artin algebra $\Lambda$ with $\operatorname{pd}_{\Lambda} M \leq$ $1, \operatorname{id}_{\Lambda} M \leq 1, \operatorname{Ext}_{\Lambda}^{1}(M, M)=0$ and with the property that if $\operatorname{Hom}_{\Lambda}(M, X) \neq$ 0 for some indecomposable $\Lambda$-module $X$ not in add $M$ then $\operatorname{Hom}_{\Lambda}(\operatorname{TrDM}, X)$ $\neq 0$. Then $M$ is a tilting and cotilting $\Lambda$-module.

Proof. Since $M$ is finitely generated, there is a map $f: \Lambda \rightarrow M^{r}$ for some $r \geq 1$ such that the induced map $\operatorname{Hom}_{\Lambda}\left(M^{r}, M\right) \rightarrow \operatorname{Hom}_{\Lambda}(\Lambda, M)$ is an epimorphism. (Note that $f: \Lambda \rightarrow M^{r}$ is a left add $M$-approximation for $\Lambda$.) Since $M$ is faithful, $f$ is a monomorphism, so that we have an exact sequence $0 \rightarrow \Lambda \rightarrow^{f}$ $M^{r} \rightarrow N \rightarrow 0$. We give the standard arguments showing that $\operatorname{pd}_{\Lambda}(M \amalg N) \leq 1$ 
and $\operatorname{Ext}_{\Lambda}^{1}(M \amalg N, M \amalg N)=0$. Since $\operatorname{Ext}_{\Lambda}^{2}(N,) \widetilde{\rightarrow} \operatorname{Ext}_{\Lambda}^{2}\left(M^{r}\right.$, ), we have $\operatorname{pd}_{\Lambda} N \leq 1$ because $\operatorname{pd}_{\Lambda} M \leq 1$. Applying $\operatorname{Hom}_{\Lambda}(, M)$ to the above exact sequence gives the exact sequence $\operatorname{Hom}_{\Lambda}\left(M^{r}, M\right) \rightarrow \operatorname{Hom}_{\Lambda}(\Lambda, M) \rightarrow$ $\operatorname{Ext}_{\Lambda}^{1}(N, M) \rightarrow \operatorname{Ext}_{\Lambda}^{1}\left(M^{r}, M\right)$ so that $\operatorname{Ext}_{\Lambda}^{1}(N, M)=0 . \operatorname{Applying}_{\operatorname{Hom}_{\Lambda}}(N$, we get an epimorphism $\operatorname{Ext}_{\Lambda}^{1}\left(N, M^{r}\right) \rightarrow \operatorname{Ext}_{\Lambda}^{1}(N, N)$, so that $\operatorname{Ext}_{\Lambda}^{1}(N, N)=0$. Applying $\operatorname{Hom}_{\Lambda}(M$,$) gives 0=\operatorname{Ext}_{\Lambda}^{1}\left(M, M^{r}\right) \rightarrow \operatorname{Ext}_{\Lambda}^{1}(M, N) \rightarrow \operatorname{Ext}_{\Lambda}^{2}(M, \Lambda)$ $=0$ exact so that $\operatorname{Ext}_{\Lambda}^{i}(M, N)=0$. From this we conclude, using the exact sequence $0 \rightarrow \Lambda \rightarrow^{f} M^{r} \rightarrow N \rightarrow 0$, that $M \coprod N$ is a tilting $\Lambda$-module.

We finally want to show that $N$ is in add $M$, so that $M$ is a tilting $\Lambda$ module. Assume to the contrary that there is an indecomposable summand $X$ of $N$ that is not in add $M$. From the exact sequence $0 \rightarrow \Lambda \rightarrow M^{r} \rightarrow N \rightarrow 0$ we have $\operatorname{Hom}_{\Lambda}(M, X) \neq 0$, and therefore it follows from our assumption that $\operatorname{Hom}_{\Lambda}(\operatorname{Tr} D M, X) \neq 0$. Since $\operatorname{id}_{\Lambda} M \leq 1$, we have $D \operatorname{Hom}_{\Lambda}(\operatorname{Tr} D M, X) \simeq$ $\operatorname{Ext}_{\Lambda}^{1}(X, M) \neq 0$ (see [R1, p. 76]). This gives the desired contradiction, and hence $M$ is a tilting module. It follows that $M$ is also a cotilting module since it has the correct number of nonisomorphic summands.

We can now prove our main result.

Theorem 1.7. Let $\Lambda$ be a finite-dimensional algebra over an algebraically closed field $k$ and let $\mathscr{C}$ be a regular component of $\Lambda$ having only a finite number of DTr-orbits and consisting entirely of modules that are not the middle of a short chain. Letting $I=\operatorname{ann} \mathscr{C}$ we have the following.

(a) $\Lambda / I$ is a tilted algebra of the form $\operatorname{End}_{H}(T)$ with $H$ a (wild) hereditary algebra and $T$ a regular tilting $H$-module.

(b) $\mathscr{C}$ is the connecting component for $\Lambda / I$.

(c) $\mathscr{C}$ is standard in $\bmod \Lambda$ and in $\bmod \Lambda / I$.

Proof. We have seen in Lemma 1.1 that the $A R$-quiver of $\mathscr{C}$ is $\mathbb{Z} \Delta$ for a finite quiver $\Delta$ without oriented cycles. Let $M$ be the direct sum of the indecomposable modules corresponding to the vertices of $\Delta$. Then by Lemma $1.2 M$ is a faithful $\Lambda / I$-module. Since $\mathscr{C}$ is standard in ind $\Lambda$ by Lemma 1.4 , we have $\operatorname{Hom}_{\Lambda / I}(M, D T r M)=0$. Then we have $\operatorname{Ext}_{\Lambda}^{1}(M, M) \simeq D \overline{\operatorname{Hom}}_{\Lambda}(M, D T r M)$ $=0$. By Lemma 1.5 and its dual, we have $\operatorname{pd}_{\Lambda / I} M \leq 1$ and, by duality, $\operatorname{id}_{\Lambda / I} M \leq 1$. Then $M$ is a tilting $\Lambda / I$-module by Lemma 1.6. Since $\mathscr{C}$ is clearly a standard component in ind $\Lambda / I$, we get that $H=\operatorname{End}_{\Lambda / I}(M)$ is the path algebra $k \Delta^{*}$, where $\Delta^{*}$ is the opposite quiver of $\Delta$. Hence $\Lambda / I=$ $\operatorname{End}_{H}(T)$ for a tilting $H$-module $T$, and so $\Lambda / I$ is a tilted algebra and $\mathscr{C}$ is a connecting component for $\Lambda / I$ (see [R2, p. 42]). Since $\mathscr{C}$ is a regular component for $\Lambda / I, T$ is a regular tilting module [R2, p. 42; R3, Theorem].

We now give some information about the modules in $\mathscr{C}$ by using reduction to tilted algebras and Theorem 1.7. As a consequence we prove the finiteness of the number of such components $\mathscr{C}$.

Lemma 1.8. Let $\Lambda=\operatorname{End}_{H}(T)$, where $H$ is a hereditary algebra and $T a$ regular tilting $H$-module. Then all but a finite number of the indecomposable modules in the connecting component $\mathscr{C}$ of $\Lambda$ are sincere.

Proof. Let $T_{1}, \ldots, T_{n}$ be all pairwise nonisomorphic indecomposable direct summands of $T$. Let $\mathscr{G}(T)$ be the full subcategory of $\bmod H$ formed by all 
modules $Y$ such that $\operatorname{Ext}_{H}^{1}(T, Y)=0$ and $\mathscr{Y}(T)$ be the full subcategory of $\bmod \Lambda$ formed by all modules $N$ such that $\operatorname{Tor}_{1}^{\Lambda}(T, N)=0$. From the theorem of Brenner and Butler we have an equivalence $F=\operatorname{Hom}_{H}(T, \quad): \mathscr{G}(T) \rightarrow$ $\mathscr{Y}(T)$ (see [R1]). Since $T$ is regular, all preinjective $H$-modules belong to $\mathscr{G}(T)$ and the image of the preinjective component of the $A R$-quiver $\Gamma_{H}$ by $F$ is a translation subquiver of $\mathscr{C}$ closed with respect to predecessors.

Let $Z$ be an indecomposable module in $\mathscr{C}$. Replacing, if necessary, $Z$ by some $(D T r)^{s} Z$, we may assume that $Z=F(X)$ for some preinjective $H$ module $X$. Clearly $P_{1}=F\left(T_{1}\right), \ldots, P_{n}=F\left(T_{n}\right)$ forms a complete set of indecomposable projective $\Lambda$-modules. For any $i, j \geq 1$ we have the isomorphisms $\operatorname{Hom}_{\Lambda}\left(P_{i},(D T r)^{j} Z\right)=\operatorname{Hom}_{\Lambda}\left(F\left(T_{i}\right), F\left((D T r)^{j} X\right)\right) \stackrel{\longrightarrow}{\rightarrow} \operatorname{Hom}_{H}\left(T_{i},(D T r)^{j} X\right)$ $\underset{\rightarrow}{\rightarrow} \operatorname{Hom}_{H}\left((\operatorname{Tr} D)^{j} T_{i}, X\right)$. Moreover,

$$
\operatorname{Ext}_{H}^{1}\left((\operatorname{Tr} D)^{j} T_{i}, X\right) \widetilde{\rightarrow} D \operatorname{Hom}_{H}\left(X,(\operatorname{Tr} D)^{j-1} T_{i}\right)=0 .
$$

By the dual of [B, Lemma 1.1 (c)] we have

$$
\operatorname{dim}_{k} \operatorname{Hom}_{H}\left((\operatorname{Tr} D)^{j} T_{i}, X\right)-\operatorname{dim}_{k} \operatorname{Ext}_{H}^{1}\left((\operatorname{Tr} D)^{j} T_{i}, X\right) \neq 0
$$

for all but a finite number of $j \geq 0$. Hence we get that $\operatorname{Hom}_{\Lambda}\left(P_{i}, D \operatorname{Tr}^{j} Z\right) \neq 0$ for all but a finite number of $j$. There is then some integer $n$ such that $(D T r)^{l} Z$ is a sincere $\Lambda$-module for all $l \geq n$. By duality there is some integer $n$ such that $(\operatorname{Tr} D)^{l} Z$ is a sincere $\Lambda$-module for all $l \geq n$. Since we have only a finite number of $D T r$-orbits, we conclude that all but a finite number of indecomposable $\Lambda$-modules in $\mathscr{C}$ are sincere.

Proposition 1.9. Let $\Lambda$ be a basic finite-dimensional algebra over an algebraically closed field $k$, and let $\mathscr{C}$ be a regular component of $\Lambda$ having only a finite number of DTr-orbits and consisting entirely of modules that are not the middle of a short chain. Write $\Lambda=P \amalg Q$ where the simple summands of $P / \operatorname{rad} P$ are exactly the composition factors of modules in $\mathscr{C}$, and denote by $\tau_{Q}(\Lambda)$ the ideal of $\Lambda$ generated by all images of all maps from $Q$ to $\Lambda$.

Then ann $\mathscr{C}=\tau_{Q}(\Lambda)$ and all but a finite number of modules in $\mathscr{C}$ are sincere $\Lambda / \tau_{Q}(\Lambda)$-modules.

Proof. It follows from Lemma 1.8 and Theorem 1.7 that all but a finite number of modules in $\mathscr{C}$ are sincere $\Lambda /$ ann $\mathscr{C}$-modules. Let $M$ be a sincere $\Lambda /$ ann $\mathscr{C}$ module in $\mathscr{C}$. Then $M$ is not the middle of a short chain in $\bmod \Lambda / \operatorname{ann} \mathscr{C}$ [RSS, Proposition 2.3] and hence is a faithful $\Lambda /$ ann $\mathscr{C}$-module by [RSS, Corollary 3.2]. Hence it follows that ann $M=\operatorname{ann} \mathscr{C}$ and ann $M=\tau_{Q}(\Lambda)$ by [RSS, Proposition 3.7].

We have the following application.

Corollary 1.10. Let $\Lambda$ be a finite-dimensional algebra over an algebraically closed field $k$. Then there is only a finite number of components $\mathscr{C}$ having only a fnite number of DTr-orbits and consisting entirely of modules which are not the middle of a short chain.

Proof. We can clearly assume that $\Lambda$ is basic and that $\mathscr{C}$ is regular since there is only a finite number of nonregular components. Then we apply Proposition 1.9 and use that there is only a finite number of ideals of the form $\tau_{Q}(\Lambda)$ when $Q$ is a projective $\Lambda$-module and that the tilted algebra $\Lambda / \tau_{Q}(\Lambda)$ has only one connecting component [R2]. 


\section{Self-injective algebras \\ WITH REGULAR COMPONENTS HAVING NO SHORT CHAINS}

Let $\Delta$ be a finite connected valued quiver with no oriented cycles and at least three vertices, and assume that $\Delta$ is neither Dynkin nor Euclidean. Then we show that there exist self-injective algebras having regular components with $A R$-quiver $\mathbb{Z} \Delta$ and consisting of nondirecting modules that are not the middle of a short chain. These self-injective algebras are associated with the tilted algebras whose regular connecting components have $A R$-quiver $\mathbb{Z} \Delta$.

For a basic connected $k$-algebra $\Lambda$ over a field $k$, let $D \Lambda=\operatorname{Hom}_{k}(\Lambda, k)$. Consider the repetitive algebra

$$
\widehat{\Lambda}=\left[\begin{array}{ccccc}
\ddots & \ddots & & & \\
& \Lambda_{i+1} & Q_{i} & & \\
& & \Lambda_{i} & Q_{i-1} & \\
& & & \Lambda_{i-1} & \ddots \\
& & & & \ddots
\end{array}\right]
$$

with $\Lambda_{i}=\Lambda$ on the main diagonal, $Q_{i}=D \Lambda$, and zeros elsewhere. The elements are all matrices with only a finite number of nonzero entries, and multiplication is given by the canonical bimodule structure of $D \Lambda$ and the zero map $D \Lambda \otimes_{\Lambda} D \Lambda \rightarrow 0$. We define for each $m \geq 1$ the algebra $R_{\Lambda}^{m}$ whose elements are the $(m+1, m+1)$ matrices

$$
\left(\begin{array}{ccccc}
\lambda_{1} & x_{1} & & 0 & \\
& \lambda_{2} & x_{2} & & \\
& & \ddots & \ddots & \\
& 0 & & \lambda_{m} & x_{m} \\
& & & & \lambda_{1}
\end{array}\right)
$$

with $\lambda_{i} \in \Lambda$ on the main diagonal and $x_{i} \in D \Lambda$. As above, the multiplication is given by the bimodule structure of $D \Lambda$ and the zero map $D \Lambda \otimes_{\Lambda} D \Lambda \rightarrow 0$. In particular, $R_{\Lambda}^{1}$ is the trivial extension $\Lambda \ltimes D \Lambda . \widehat{\Lambda}$ and $R_{\Lambda}^{m}$ will also denote the associated $k$-categories whose objects are representatives of the isomorphism classes of indecomposable projective modules. The identity maps $A_{i} \rightarrow A_{i+1}$ and $Q_{i} \rightarrow Q_{i+1}$ induce an automorphism $\nu_{\Lambda}$ of $\widehat{\Lambda}$, called the Nakayama automorphism. We denote by $\nu_{\Lambda}$ also the induced automorphism $\bmod \hat{\Lambda} \rightarrow$ $\bmod \hat{\Lambda}$, where $\bmod \hat{\Lambda}$ is the category of finite-dimensional $\hat{\Lambda}$-modules. The category $R_{\Lambda}^{m}$ is just the quotient category $\widehat{\Lambda} /\left(\nu_{\Lambda}^{m}\right)$.

Before we give the main result of this section we prove some general lemmas.

Lemma 2.1. Let $\Lambda$ be a self-injective artin algebra. Then every indecomposable $\Lambda$-module is nondirecting.

Proof. Let $M$ be an indecomposable $\Lambda$-module, and let $S$ be a simple composition factor of $M, P$ the projective cover of $S$, and $I$ the injective envelope of $S$. Then $\operatorname{Hom}_{\Lambda}(P, M) \neq 0, \operatorname{Hom}_{\Lambda}(M, I) \neq 0$, and $I=\nu P$, where $\nu$ is the Nakayama automorphism of $\Lambda$. Since $P \simeq \nu^{i} P$ for some $i \geq 1$, we have an oriented cycle of nonzero maps $P \rightarrow M \rightarrow \nu P \rightarrow \nu^{2} P \rightarrow \cdots \rightarrow \nu^{i} P \simeq P$.

Lemma 2.2. Let $B$ be an artin algebra with ind $B=\mathscr{X} \vee \mathscr{C} \vee \mathscr{Y}$ where $\mathscr{C}$ is a component, $\mathscr{X}$ is a union of components containing all projective modules, $\mathscr{Y}$ 
is a union of components containing all injective modules, and $\operatorname{Hom}_{B}(\mathscr{C}, \mathscr{X})=$ $0=\operatorname{Hom}_{B}(\mathscr{Y}, \mathscr{X} \vee \mathscr{C})$. Then we have ind $\widehat{B}=\bigvee_{p \in Z}\left(\mathscr{C}_{p} \vee \mathscr{L}_{p}\right)$, where the $\mathscr{C}_{p}$ and $\mathscr{L}_{p}$ satisfy the following properties:

(a) $\mathscr{C}_{p}=\nu_{B}^{p} \mathscr{C}_{0}$ for all $p \in Z$ and is a component isomorphic to $\mathscr{C}$, and $\mathscr{L}_{p}=\nu_{B}^{p} \mathscr{L}_{0}$ is a union of components.

(b) $\operatorname{Hom}_{\widehat{B}}\left(\mathscr{L}_{p}, \mathscr{C}_{q}\right) \neq 0$ implies $q=p+1$.

(c) $\operatorname{Hom}_{\widehat{B}}\left(\mathscr{C}_{p}, \mathscr{C}_{q} \vee \mathscr{L}_{q}\right) \neq 0$ implies $q=p$.

Proof. We identify $\bmod B$ with the subcategory $\bmod B_{0}$ of $\bmod \widehat{B}$. Hence we have, by assumption, ind $B_{0}=\mathscr{X}_{0} \vee \mathscr{C}_{0} \vee \mathscr{Y}_{0}$ satisfying the properties in the statement of the lemma. We first want to show that ind $\widehat{B}=\mathscr{X}_{0}^{\prime} \vee \mathscr{C}_{0} \vee \mathscr{Y}_{0}^{\prime}$, where $\mathscr{X}_{0}^{\prime}$ is a union of components where the simple composition factors are of the form $\nu_{B}^{t} S$ for $t \leq 0$ and $S$ a simple $B_{0}$-module and $\mathscr{Y}_{0}^{\prime}$ is a union of components where the simple composition factors are of the form $\nu_{B}^{t} S$ for $t \geq 0$ and $S$ a simple $B_{0}$-module. Further we claim that $\operatorname{Hom}_{\widehat{B}}\left(\mathscr{C}_{0}, \mathscr{X}_{0}^{\prime}\right)=$ $0=\operatorname{Hom}_{\widehat{B}}\left(\mathscr{Y}_{0}^{\prime}, \mathscr{X}_{0}^{\prime} \vee \mathscr{C}_{0}\right)$.

To see this, consider first the natural embedding $\bmod B \subset \bmod A_{1}$, where $A_{1}=\left(\begin{array}{cc}B_{1} & D B \\ 0 & B_{0}\end{array}\right)$. We want to show that $\mathscr{C}_{0}$ remains a component and $\mathscr{X}_{0}$ remains a union of components in $\bmod A_{1}$. For this it is sufficient to show that an indecomposable $A_{1}$-module that is not a $B_{0}$-module has no nonzero maps to modules in $\mathscr{Z}_{0} \vee \mathscr{C}_{0}$. Each $A_{1}$-module can be viewed as a triple $\left(U_{B_{1}}, V_{B_{0}}, f\right)$ where $f: U \otimes_{B_{1}} D B \rightarrow V_{B_{0}}$. Let $\left(0, W_{b_{0}}, 0\right)$ be in $\bmod B_{0}$, and let $(0, h)$ be a nonzero map from an indecomposable $A_{1}$-module $\left(U_{B_{1}}, V_{B_{0}}, f\right)$ not in mod $B_{0}$ to an indecomposable $A_{1}$-module $\left(0, W_{B_{0}}, 0\right)$. Then $h: V_{B_{0}} \rightarrow W_{B_{0}}$ is nonzero, and since $\left(U_{B_{1}}, V_{B_{0}}, f\right)$ is not in $\bmod B_{0}, f: U \otimes_{B_{1}} D B \rightarrow V_{B_{0}}$ is nonzero. Considering an epimorphism $B_{1}^{t} \rightarrow U$, we get an epimorphism $B_{1}^{t} \otimes_{B_{1}} D B \simeq(D B)_{B_{0}}^{t} \rightarrow U \otimes_{B_{1}} D B$. This shows that all indecomposable summands of $V_{B_{0}}$ are in $\mathscr{Y}_{0}$ and hence $W_{B_{0}}$ is in $\mathscr{Y}_{0}$.

We continue by passing from $A_{1}$ to

$$
\left(\begin{array}{ccc}
B_{2} & D B & 0 \\
0 & B_{1} & D B \\
0 & 0 & B_{0}
\end{array}\right)=\left(\begin{array}{cc}
B_{2} & D B \\
0 & A_{1}
\end{array}\right)
$$

etc., and it is easy to see by using the triple description of $A_{2}$-modules that $\mathscr{X}_{0}$ and $\mathscr{C}_{0}$ still remain unchanged. Writing

$$
A=\left(\begin{array}{llll}
\ddots & \ddots & & \\
& B_{2} & D B & \\
& & B_{1} & D B \\
& & & B_{0}
\end{array}\right)
$$

we get ind $A=\mathscr{X}_{0} \vee \mathscr{C}_{0} \vee \mathscr{Y}_{0}^{\prime}$ and $\operatorname{Hom}_{A}\left(\mathscr{Y}_{0}^{\prime}, \mathscr{X}_{0} \vee \mathscr{C}_{0}\right)=0$.

We next want to pass from $A$ to $C_{1}=\left(\begin{array}{ll}A & D B \\ 0 & B_{-1}\end{array}\right)$. Here it is convenient to view the $C_{1}$-modules as triples $\left(U_{A}, V_{B_{-1}}, g\right), g: U_{A} \rightarrow \operatorname{Hom}_{B_{-1}}\left(D B, V_{B_{-1}}\right)$. We now want to show that $\mathscr{C}_{0}$ and $\mathscr{Y}_{0}^{\prime}$ remain unchanged by showing that there is no nonzero map from $\mathscr{C}_{0} \vee \mathscr{Y}_{0}^{\prime}$ to any indecomposable $C_{1}$-module that is not an $A$-module. For this we consider a monomorphism $V_{B_{-1}} \rightarrow(D B)^{t}$ and the induced monomorphism $\operatorname{Hom}_{B_{-1}}\left(D B, V_{B_{-1}}\right) \rightarrow \operatorname{Hom}_{B_{-1}}\left(D B,(D B)^{t}\right) \simeq B_{0}^{t}$. It 
follows that if we have a nonzero map $(h, 0)$ from an indecomposable $C_{1}$ module $\left(W_{A}, 0,0\right)$ to some indecomposable $\left(U_{A}, V_{B_{-1}}, g\right)$ not in $\bmod A$, then $W_{A}$ must be in $\mathscr{Z}_{0}$. Continuing this process, we get ind $\widehat{B}=\mathscr{Q}_{0}^{\prime} \vee \mathscr{C}_{0} \vee \mathscr{Y}_{0}^{\prime}$ with $\operatorname{Hom}_{\widehat{B}}\left(\mathscr{C}_{0}, \mathscr{X}_{0}^{\prime}\right)=0=\operatorname{Hom}\left(\mathscr{Y}_{0}^{\prime}, \mathscr{X}_{0}^{\prime} \vee \mathscr{C}_{0}\right)$. Also note that it follows from the construction that the simple composition factors of $\mathscr{Y}_{0}^{\prime}$ are of the form $\nu_{B}^{t} S$ for $t \geq 0$ and $S$ a simple $B_{0}$-module and the simple composition factors of $\mathscr{X}_{0}^{\prime}$ of the form $\nu_{B}^{t} S$ for $t \geq 0$ and a simple $B_{0}$-module $S$.

Applying $\nu_{B}^{p}$ we get ind $\widehat{B}=\mathscr{X}_{p}^{\prime} \vee \mathscr{C}_{p} \vee \mathscr{Y}_{p}$, where $\mathscr{X}_{p}^{\prime}=\nu_{B}^{p} \mathscr{X}, \mathscr{C}_{p}=\nu_{B}^{p} \mathscr{C}_{0}$, and $\mathscr{Y}_{p}=\nu_{B}^{p} \mathscr{Y}_{0}$. Here the simple composition factors in $\mathscr{C}_{p}$ are of the form $\nu_{B}^{p} S$, in $\mathscr{Y}_{p}^{\prime}$ of the form $\nu_{B}^{t} S$ for $t \geq p$, and in $\mathscr{X}_{p}^{\prime}$ of the form $\nu_{B}^{t} S$ for $t \leq p$. Denote by $\mathscr{L}_{p}$ the full subcategory of ind $\widehat{B}$ whose objects have simple composition factors of the form $\nu_{B}^{p} S$ and $\nu_{B}^{p+1} S$, but are not in $\mathscr{C}_{p} \vee \mathscr{C}_{p+1}$. Then it follows that each indecomposable $\widehat{B}$-module is in some $\mathscr{C}_{p}$ or $\mathscr{L}_{p}$ and that $\mathscr{L}_{p}$ is a union of components. The rest of the claims are now easy consequences.

Theorem 2.3. Let $H$ be a hereditary basic connected algebra of type $\Delta$ over an algebraically closed field $k$, where $\Delta$ has at least three vertices and is neither of Dynkin nor Euclidean type. Let $T$ be a regular tilting module and $B=$ $\operatorname{End}_{H}(T)$. Then we have

(a) For $m \geq 2$, the AR-quiver of $R_{B}^{m}$ contains at least $m$ regular components of the form $\mathbb{Z} \Delta^{*}$ (where $\Delta^{*}$ denotes the opposite quiver of $\Delta$ ) and consists entirely of nondirecting modules that are not the middle of a short chain.

(b) The AR-quiver of $R_{B}^{1}=B \ltimes D B$ has a regular component of the form $\mathbb{Z} \Delta^{*}$ and consists entirely of modules that are the middle of a short chain.

Proof. For a fixed $m \geq 1$ consider the canonical Galois covering $F^{m}: \widehat{B} \rightarrow$ $\widehat{B} /\left(\nu_{B}^{m}\right)=R_{B}^{m}$ and the associated pushdown functor $F_{\lambda}^{m}: \bmod \widehat{B} \rightarrow \bmod R_{B}^{m}$ [BG]. Since $\left(\nu_{B}^{m}\right)$, as a torsionfree group, acts freely on the isomorphism classes of indecomposable objects in $\bmod \widehat{B}, F_{\lambda}^{m}$ preserves indecomposable modules and almost split sequences [G2]. $\widehat{B}$ is locally support-finite by [S1,R3]; that is, for each indecomposable projective module in $\bmod \widehat{B}$ there are only finitely many nonisomorphic indecomposable projective modules $P^{\prime}$ in $\bmod \widehat{B}$ such that $\operatorname{Hom}_{\widehat{B}}(P, X) \neq 0$ and $\operatorname{Hom}_{\widehat{B}}\left(P^{\prime}, X\right) \neq 0$ for some indecomposable module $X$ in $\bmod \widehat{B}$. Then $F_{\lambda}^{m}$ is dense by [DLS] and consequently, we have a Galois covering $F_{\lambda}^{m}: \bmod \widehat{B} \rightarrow \bmod R_{B}^{m}$. In particular, the $A R$-quiver of $R_{B}^{m}$ coincides with the orbit space $\Gamma_{\widehat{B}} /\left(\nu_{B}^{m}\right)$. Hence the images $\mathscr{C}_{0}^{\prime}=F_{\lambda}^{m}\left(\mathscr{C}_{0}\right)=$ $F_{\lambda}^{m}\left(\mathscr{C}_{m}\right), \mathscr{C}_{1}^{\prime}=F_{\lambda}^{m}\left(\mathscr{C}_{1}\right), \ldots, \mathscr{C}_{m-1}^{\prime}=F_{\lambda}^{m}\left(\mathscr{C}_{m-1}\right)$ of $\mathscr{C}_{0}, \ldots, \mathscr{C}_{m-1}$ by $F_{\lambda}^{m}$ are regular components of $\Gamma_{R_{B}^{m}}$ and of the form $\mathbb{Z} \Delta^{*}$.

We now show that for $m \geq 2$ all modules in $\mathscr{C}_{0}^{\prime}, \ldots, \mathscr{C}_{m-1}^{\prime}$ are not the middle of a short chain. We assume without loss of generality that $M$ is in $\mathscr{C}_{0}^{\prime}$, and since $F_{\lambda}^{m}$ is dense, we have $M=F_{\lambda}^{m} N$ for some $N$ in $\mathscr{C}_{0}$. Suppose that $X \rightarrow M \rightarrow D T r X$ is a short chain in $\bmod R_{B}^{m}$. Since $F_{\lambda}^{m}: \bmod \widehat{B} \rightarrow$ $\bmod R_{B}^{m}$ is a Galois covering, there exists an indecomposable $\widehat{B}$-module $Y$ such that $F_{\lambda}^{m} Y=X$ and $\operatorname{Hom}_{\widehat{B}}(N, D \operatorname{Tr} Y) \neq 0$. Since $B$ is a tilted algebra via a regular tilting module, the assumption of Lemma 2.2 is satisfied with $\mathscr{C}$ the connecting component [R2]. It then follows by Lemma 2.2 that $D \operatorname{Tr} Y$, 
and hence also $Y$, must be in $\mathscr{C}_{0}$ or $\mathscr{L}_{0}$. Since the connecting component $\mathscr{C}$ for the tilted algebra $B$ is directing and $\mathscr{C}$ is isomorphic to $\mathscr{C}_{0}$ by Lemma 2.2, DTrY and $Y$ cannot be in $\mathscr{C}_{0}$. But $\operatorname{Hom}_{\widehat{B}}\left(\mathscr{L}_{0}, \nu_{B}^{r m} \mathscr{C}_{0}\right)=0$ for all $r \in Z$ when $m \geq 2$, by Lemma 2.2 , so that $\operatorname{Hom}_{B}(X, M)=0$. This contradiction shows that $M$ is not the middle of a short chain. That all modules in $\mathscr{C}_{0}$ are nondirecting follows by Lemma 2.1 .

Assume now that $m=1$. Then $R_{B}^{1}=B \ltimes D B$ is a symmetric algebra, and hence for any indecomposable $R_{B}^{1}$-module $M$ we have a short chain $P / S \rightarrow$ $M \rightarrow D T r(P / S)=\operatorname{rad} P$, where $S$ is a simple summand of the socle of $M$ and $P$ is its projective cover. In particular, all modules in $\mathscr{C}_{0}^{\prime}$ are the middle of a short chain.

\section{REFERENCES}

[A] M. Auslander, Applications of morphisms determined by objects, Lecture Notes in Pure and Appl. Math., vol. 37, Dekker, New York, 1976, pp. 245-327.

[AR] M. Auslander and I. Reiten, Modules determined by their composition factors, Illinois J. Math. 29 (1985), 280-301.

[B] D. Baer, Wild hereditary artin algebras and linear methods, Manuscripta Math. 55 (1986), 69-82.

[BG] K. Bongartz and P. Gabriel, Covering spaces in representation theory, Invent. Math. 65 (1981), 331-378.

[DLS] P. Dowbor, A. Lenzing, and A. Skowroński, Galois coverings of algebras by locally supportfinite categories, Representation Theory I. Finite Dimensional Algebras, Lecture Notes in Math., vol. 1177, Springer-Verlag, Berlin and New York, 1986, pp. 91-93.

[DR] V. Dlab and C. M. Ringel, Indecomposable representations of graphs and algebras, Mem. Amer. Math. Soc., vol. 173, Amer. Math. Soc., Providence, RI, 1976.

[G1] P. Gabriel, Auslander-Reiten sequences and representation-finite algebras, Representation Theory I, Lectures Notes in Math., vol. 831, Springer-Verlag, Berlin and New York, 1980, pp. $1-71$.

[G2] _ The universal cover of a representation-finite algebra, Representations of Algebras, Lecture Notes in Math., vol. 903, Springer-Verlag, Berlin and New York, 1981, pp. 68-105.

[HW] D. Hughes and J. Waschbüsch, Trivial extensions of tilted algebras, Proc. London Math. Soc. (3) 46 (1983), 347-364.

[H] D. Happel, On the derived category of a finite dimensional algebra, Comment. Math. Helv. 62 (1987), 339-389.

[IT] K. Igusa and G. Todorov, Radical layers of representable functors, J. Algebra 89 (1984), $105-146$.

[L] S. Liu, Degrees of irreducible maps and Auslander-Reiten quivers, J. London Math. Soc. (to appear).

[RSS] I. Reiten, A. Skowroński, and S. O. Smalø, Short chains and short cycles of modules, Proc. Amer. Math. Soc., this issue.

[R1] C. M. Ringel, Tame algebras and integral quadratic forms, Lecture Notes in Math., vol. 1099, Springer-Verlag, Berlin and New York, 1984.

[R2] _ Representation theory of finite dimensional algebras, Representations of Algebras, London Math. Soc. Lecture Notes Ser., vol. M6, Cambridge Univ. Press, Cambridge, 1986, pp. 7-79.

[R3] _ The regular components of the Auslander-Reiten quiver of a tilted algebra, Chinese Ann. Math. Ser. B 1 (1988), 1-18.

[S1] A. Skowroński, A characterization of a new class of artin algebras, J. London Math. Soc. (3) 26 (1982), 53-63. 
[S2] - Generalization of Yamagata's theorem on trivial extensions, Arch. Math. 48 (1987), 68-76.

[SiSk] D. Simson and A. Skowroński, Extension of artinian rings by hereditary injective modules, Representations of Algebras, Lecture Notes in Math., vol. 903, Springer-Verlag, Berlin and New York, 1981, pp. 315-330.

[SkSm] A. Skowroński and S. O. Smalø, Directing modules, J. Algebra 147 (1992), 137-146.

[T] H. Tachikawa, Representations of trivial extensions of hereditary algebras, Representation Theory II, Lecture Notes on Math., vol. 832, Springer-Verlag, Berlin and New York, 1980, pp. 579-599.

[Z] Y. Zhang, The structure of regular components, Canad. J. Math. (to appear).

Department of Mathematics and Statistics, University of Trondheim, AVH, 7055, DragVoll, NorwaY

E-mail address: Idun.Reiten@avh.unit.no

Mathematical Institute, Nicholas Copernicus University, Chopina 12/18, 87-100, TORUn, Poland

E-mail address: Skowron@pltumk11.bitnet

Department of Mathematics and Statistics, University of Trondheim, AVH, 7055, Dragvoll, Poland

E-mail address: Sverre.Smalo@avh.unit.no 